\title{
A class of nonlinear wave equations containing the continuous Toda case
}

\author{
E. Alfinito ${ }^{c, b}$, M.S. Causo ${ }^{a, b}$, G. Profilo $^{d}$, and G. Soliani ${ }^{a, b}$. \\ ${ }^{a}$ Dipartimento di Fisica dell'Università, Lecce, Italy \\ ${ }^{b}$ Istituto Nazionale di Fisica Nucleare, Sezione di Lecce, Italy, \\ ${ }^{c}$ Dipartimento di Fisica dell'Università, Salerno, Italy, \\ ${ }^{d}$ Dipartimento di Matematica dell'Università, Lecce, Italy.
}

\begin{abstract}
We consider a nonlinear field equation which can be derived from a binomial lattice as a continuous limit. This equation, containing a perturbative friction-like term and a free parameter $\gamma$, reproduces the Toda case (in absence of the friction-like term) and other equations of physical interest, by choosing particular values of $\gamma$. We apply the symmetry and the approximate symmetry approach, and the prolongation technique. Our main purpose is to check the limits of validity of different analytical methods in the study of nonlinear field equations. We show that the equation under investigation with the friction-like term is characterized by a finite-dimensional Lie algebra admitting a realization in terms of boson annhilation and creation operators. In absence of the friction-like term, the equation is linearized and connected with equations of the Bessel type. Examples of exact solutions are displayed, and the algebraic structure of the equation is discussed.
\end{abstract}




\section{Introduction}

In this paper we study the class of nonlinear wave equations

$$
u_{t t}+\epsilon u_{t}=\left[\left(1+\frac{u}{\gamma}\right)^{\gamma-1}\right]_{x x}
$$

where $u=u(x, t)$, subscripts denote partial derivatives, $\epsilon$ is a free parameter, and $\gamma$ is a constant which takes all (real) values except zero and one.

For $\epsilon=0$, Eq.(1.1) can be considered as the continuous limit of a uniform onedimensional nonlinear lattice of $N$ particles interacting through the nearest-neighbour potential [1]

$$
\phi\left(r_{n}\right)=\frac{a_{n}}{b_{n}}\left[\left(1+\frac{b_{n} r_{n}}{\gamma}\right)^{\gamma}-\left(1+b_{n} r_{n}\right)\right]
$$

where $a_{n}$ and $b_{n}$ are constants of the $n$-th nonlinear spring.

The equation of motion of the chain reads

$$
M_{n} \ddot{y}_{n}=-\left[\phi^{\prime}\left(r_{n+1}\right)-\phi^{\prime}\left(r_{n}\right)\right]
$$

where $\phi^{\prime}=\frac{d \phi}{d r}, M_{n}$ is the mass of the $n$-particle and $r_{n}=y_{n}-y_{n+1}, y_{n}$ being the displacement of the $n$-particle from its the equilibrium position. We shall call "binomial lattice" the chain governed by the potential (1.2). If the binomial lattice is uniform, i.e. $a_{n}=a, b_{n}=b$ and $M_{n}=M$ for any $n$, the equation of motion (1.3) takes the form

$$
\ddot{r}_{n}=\frac{a}{M}\left[\left(1+\frac{b}{\gamma} r_{n+2}\right)^{\gamma-1}-2\left(1+\frac{b}{\gamma} r_{n+1}\right)^{\gamma-1}+\left(1+\frac{b}{\gamma} r_{n}\right)^{\gamma-1}\right] .
$$

For $\gamma \rightarrow \infty, \gamma=2, \gamma=3$ and $\gamma=-1$, Eq.(1.4) reproduces the Toda [2], the harmonic, the Fermi-Pasta-Ulam [3] and a Coulomb-like uniform lattice, respectively. Since Eq.(1.4) models interesting physical situations, its analysis may be important mostly from the point of view of a unifying theory of lattice systems described by the potential (1.2).

This difficult task is made simpler by starting from the investigation of the continuous limit of Eq.(1.4). Then, Eq.(1.4) becomes

$$
u_{t t}=\left[\left(1+\frac{u}{\gamma}\right)^{\gamma-1}\right]_{x x}
$$

after the rescaling $b r \rightarrow u$ and $\sqrt{\frac{a b}{M}} t \rightarrow t$. 
For $\gamma \rightarrow \infty$, Eq.(1.5) coincides with the $(1+1)$-dimensional continuous Toda equation 《4

$$
u_{t t}=\left(e^{u}\right)_{x x},
$$

which is known also as heavenly equation and plays a crucial role in General Relativity [5], [6].

Equation (1.1) represents a modified version of Eq.(1.5). It contains a friction-like term which can be considered as a small perturbation. This extension of Eq.(1.5) allows us to check the validity of different analytical methods usually employed to handle integrable equations.

Precisely, here we apply to Eq.(1.1) the symmetry [7] and the approximate symmetry approach [8], and the prolongation procedure [9].

The main results achieved in this paper are the following. In Sec. 2 we find the approximate symmetry generators of Eq.(1.1) in the case in which $\epsilon$ is a perturbative parameter. The commutation relations among these operators do not define an exact finite-dimensional algebra. (Exact algebras arise only for $\epsilon=0$ and when $\epsilon \neq 0$ is not considered as a small quantity). Notwithstanding, an exact finite-dimensional Lie algebra can be constructed by introducing certain auxiliary operators. A realization of this algebra, which characterizes the approximate symmetries of Eq. (1.1), is obtained in terms of boson annihilation and creation operators.

In Sec. 3, the symmetry approach is exploited to determine special solutions of Eq.(1.1) and Eq.(1.5). In this context, the study of the symmetry corresponding to the generator $X^{0}{ }_{4}$ (see $(2.5 \mathrm{c})$ ) is of particular interest. In fact, $X^{0}{ }_{4}$ is a scale symmetry in the variables $x, u$ together with a translation of $\frac{2 \gamma}{\gamma-2}$ along $u$. For $\gamma \rightarrow \infty$, i.e. when Eq.(1.1) coincides with the continuous Toda equation, the scale symmetry is broken. The reduced equation related to $X^{0}{ }_{4}$ resembles the one-dimensional Liouville equation (see (3.4)) containing a square derivative which vanishes in the limit $\gamma \rightarrow \infty$. In the case $\epsilon=0$, Eq.(3.4) is exactly solved for some values of the parameter $\gamma$. For example, if $\gamma=3$ (a choice associated with the Fermi-Pasta-Ulam potential), we get a solution to Eq.(1.5) via Eq.(3.4) expressed in terms of the Weierstrass function.

For $\epsilon \neq 0$, we provide an implicit solution to Eq.(1.1) by using the symmetry variable $V=v \partial_{x}+\partial_{t}$, where $v$ is a constant. This solution (see (3.42)) involves a generalization of the exponential-integral function. It is notable that for $\gamma \rightarrow \infty$, this solution reproduces just that corresponding to the continuous Toda case [10].

Sec. 4 is devoted to a study of Eq.(1.1) within the prolongation scheme [9]. This method is particularly convenient because it allows us to analyze Eq.(1.1) for $\epsilon=0$ and $\epsilon \neq 0$ by an algebraic point of view. We show that the (differential) prolongation equations for Eq.(1.5) afford a class of solutions connected with the Lie algebra of the Euclidean group $E_{2}$ in the plane. This algebra leads to a linearized version of Eq.(1.5), in the sense that a mapping is established between Eq.(1.5) and the linear wave equation (4.6) written in a pseudopotential variable. For $\gamma \rightarrow \infty$, Eq.(4.6) coincides with a two-dimensional form of a linear wave equation that occurs in a 
generalized Gibbson-Hawking ansatz [11]. On the other hand, the prolongation equations for Eq.(1.1) can be solved in terms of power series expansions whose coef-

ficients (in the variable $z=\left(1+\frac{u}{\gamma}\right)^{\gamma-1}$ ) depend on the pseudopotential components and, for a certain infinite set of values of $\gamma$, obey a finite-dimensional Lie algebra. A representation of this algebra indicates the existence of a possible link between the prolongation method and the symmetry approach. Finally, in Sec. 5, some comments are reperted.

\section{The approximate symmetry approach}

In the case in which $\epsilon$ is a perturbative parameter, Eq.(1.1) can be handled profitably by means of the method devised by Baikov, Gazizov and Ibragimov some years ago [8]. This method enables one to construct approximate symmetries which are stable for small perturbations of the differential equation under investigation.

In order to obtain the approximate symmetries of Eq. $(1.1)(\epsilon \neq 0)$, we have to introduce the quantities

$$
\begin{aligned}
& F_{0}=u_{t t}-\left[(1+u / \gamma)^{\gamma-1}\right]_{x x}, \\
& F_{1}=u_{t}
\end{aligned}
$$

into the equations (3.16) and (3.17) of [8].

For brevity, we shall omit any calculation and report below the results only.

The approximate symmetry generator turns out to be

$$
\begin{aligned}
X= & {\left[c_{1} t+c_{2}+\epsilon\left(\frac{\gamma-2}{\gamma+2} c_{1} \frac{t^{2}}{2}+k_{1} t+k_{2}\right)\right] \partial_{t}+\left[\left(c_{1}+c_{2}\right) x+c_{4}+\epsilon\left(\left(k_{1}+k_{3}\right) x+k_{4}\right)\right] \partial_{x} } \\
& +\left[\frac{2 \gamma}{\gamma-2} c_{3}\left(1+\frac{u}{\gamma}\right)+2 \epsilon \frac{\gamma}{\gamma-2}\left(k_{3}-\frac{\gamma-2}{\gamma+2} c_{1} t\right)\left(1+\frac{u}{\gamma}\right)\right] \partial_{u}
\end{aligned}
$$

with $c_{1}, c_{2}, \ldots$ and $k_{1}, k_{2}, \ldots$ arbitrary constants.

From (2.2) we get

$$
X_{1}=X_{1}^{0}+\epsilon\left[\frac{\gamma-2}{\gamma+2} \frac{t^{2}}{2} \partial_{t}-\frac{2 \gamma t}{\gamma+2}\left(1+\frac{u}{\gamma}\right) \partial_{u}\right]
$$

where

$$
\begin{gathered}
X_{1}^{0}=t \partial_{t}+x \partial_{x}, \\
X_{2} \equiv X_{2}^{0}=\partial_{t} \\
X_{3} \equiv X_{3}^{0}=\partial_{x}
\end{gathered}
$$




$$
\begin{aligned}
& X_{4} \equiv X_{4}^{0}=x \partial_{x}+\frac{2 \gamma}{\gamma-2}\left(1+\frac{u}{\gamma}\right) \partial_{u}, \\
& X_{5}=\epsilon\left(t \partial_{t}+x \partial_{x}\right) \\
& X_{6}=\epsilon \partial_{t}, \\
& X_{7}=\epsilon\left[x \partial_{x}+\frac{2 \gamma}{\gamma-2}\left(1+\frac{u}{\gamma}\right) \partial_{u}\right], \\
& X_{8}=\epsilon \partial_{x} .
\end{aligned}
$$

The operators $X_{1}^{0}, X_{2}^{0}, X_{3}^{0}, X_{4}^{0}$ are the (exact) symmetry generators of Eq. (1.1) for $\epsilon=0$, while $X_{1}, X_{2}, X_{3}, X_{4}$ are the approximate symmetry generators of Eq. (1.1) for $\epsilon \neq 0$. The exact symmetry generators of Eq.(1.1) for $\epsilon \neq 0$ are $X_{1}^{0}, X_{2}^{0}$ and $X_{3}^{0}$. The operators $X_{5}^{0}, X_{6}^{0}, X_{7}^{0}, X_{8}^{0}$ are inessential.

The approximate symmetry generators $X_{1}, X_{2}, X_{3}, X_{4}$ satisfy the commutation relations

$$
\begin{aligned}
& {\left[X_{2}, X_{1}\right]=X_{2}+\epsilon\left[\frac{\gamma-2}{\gamma+2} t \partial_{t}-\frac{2 \gamma}{\gamma+2}\left(1+\frac{u}{\gamma}\right) \partial_{u}\right],} \\
& {\left[X_{2}, X_{4}\right]=0, \quad\left[X_{2}, X_{3}\right]=0, \quad\left[X_{1}, X_{4}\right]=0,} \\
& {\left[X_{1}, X_{3}\right]=-X_{3}, \quad\left[X_{3}, X_{4}\right]=X_{3}}
\end{aligned}
$$

The commutation rules (2.6)-(2.8) define an exact finite-dimensional Lie algebra only for $\epsilon=0$. However, they can be exploited to build up a realization of an exact Lie algebra, which holds for $\epsilon \neq 0$, with the help of the "auxiliary" operators

$$
\begin{aligned}
& Z=\frac{\gamma-2}{\gamma+2} t \partial_{t}-\frac{2 \gamma}{\gamma+2}\left(1+\frac{u}{\gamma}\right) \partial_{u} \\
& Y=\frac{\gamma-2}{\gamma+2} \frac{t^{2}}{2} \partial_{t}-\frac{2 \gamma t}{\gamma+2}\left(1+\frac{u}{\gamma}\right) \partial_{u}
\end{aligned}
$$

In doing so, we find

$$
\begin{aligned}
& {\left[X_{2}, X_{1}\right]=X_{2}+\epsilon Z} \\
& {\left[X_{2}, X_{4}\right]=\left[X_{2}, X_{3}\right]=\left[X_{1}, X_{4}\right]=0,} \\
& {\left[X_{1}, X_{3}\right]=-X_{3}, \quad\left[X_{3}, X_{4}\right]=X_{3},} \\
& {\left[X_{1}, Z\right]=-\epsilon \frac{\gamma-2}{\gamma+2} Y, \quad\left[X_{1}, Y\right]=Y,} \\
& {\left[X_{2}, Y\right]=Z, \quad\left[X_{2}, Z\right]=\frac{\gamma-2}{\gamma+2} X_{2}, \quad[Z, Y]=\frac{\gamma-2}{\gamma+2} Y,} \\
& {\left[X_{3}, Y\right]=\left[X_{3}, Z\right]=\left[X_{4}, Y\right]=\left[X_{4}, Z\right]=0 .}
\end{aligned}
$$


Hereafter, the symbols $Y, Z, X_{j}(j=1, . ., 4)$ will mean both the abstract elements and the corresponding realizations (see (2.5), (2.9) and (2.10)) of the finitedimensional Lie algebra $(2.10)$.

At this stage, we remark that the subalgebra (2.10e) is isomorphic to the $\operatorname{sl}(2, R)$ algebra

$$
\left[Z^{\prime}, T\right]=2 S, \quad[T, S]=2 Z^{\prime}, \quad\left[S, Z^{\prime}\right]=-2 T
$$

where

$$
\begin{aligned}
T & =\sqrt{2 \frac{\gamma+2}{\gamma-2}}\left(Y+X_{2}\right), \quad S=\sqrt{2 \frac{\gamma+2}{\gamma-2}}\left(Y-X_{2}\right), \\
Z^{\prime} & =2 \frac{\gamma+2}{\gamma-2} Z
\end{aligned}
$$

It can be proved straightforwardly that

Proposition 1 The Casimir operator

$$
\begin{aligned}
C & =T^{2}-S^{2}-Z^{\prime 2} \\
& \equiv 4 \frac{\gamma+2}{\gamma-2}\left[2 X_{2} Y-Z\left(\frac{\gamma+2}{\gamma-2} Z+1\right)\right]
\end{aligned}
$$

of the Lie algebra (2.11), commutes with all the elements $Y, Z, X_{j}(j=1, . ., 4)$ of the Lie algebra (2.10).

Furthermore, the following Proposition holds:

Proposition 2 The Lie algebra (2.10) admits a realization in terms of boson annihilation and creation operators.

This can be seen by setting

$$
a_{1}^{+}=t, \quad a_{2}^{+}=u, \quad a_{3}^{+}=x, \quad a_{1}=\partial_{t}, \quad a_{2}=\partial_{u}, \quad a_{3}=\partial_{x},
$$

to give

$$
\begin{aligned}
{\left[a_{j}, a_{k}^{+}\right] } & =\delta_{j k}, \quad\left[a_{j}, a_{k}\right]=0, \quad\left[a_{j}^{+}, a_{k}^{+}\right]=0, \quad(j, k=1,2,3), \\
Y & =\frac{\gamma-2}{2(\gamma+2)} a_{1}^{+2} a_{1}-\frac{2 \gamma a_{1}^{+}}{\gamma+2}\left(1+\frac{1}{\gamma} a_{2}^{+}\right) a_{2}, \\
Z & =\frac{\gamma-2}{\gamma+2} a_{1}^{+} a_{1}-\frac{2 \gamma}{\gamma+2}\left(1+\frac{1}{\gamma} a_{2}^{+}\right) a_{2}, \\
X_{1} & =a_{1}^{+} a_{1}+a_{3}^{+} a_{3}+\epsilon Y, \quad X_{2}=a_{1}, \quad X_{3}=a_{2}, \\
X_{4} & =a_{3}^{+} a_{3}+\frac{2 \gamma}{\gamma-2}\left(1+\frac{1}{\gamma} a_{2}^{+}\right) a_{2},
\end{aligned}
$$


and

$$
C=\left(\frac{\gamma}{\gamma-2}\right)^{2} C_{\infty}+C(\gamma)
$$

where $C_{\infty}=-8 a_{2}\left(2 a_{2}+1\right)$ is the Casimir invariant relative to the continuous Toda case $\gamma \rightarrow \infty$, and the operator $C(\gamma)$, defined by

$$
C(\gamma)=-\frac{8 a_{2}^{+} a_{2}}{\gamma-2}\left[1+\frac{2 \gamma}{\gamma-2}\left(2 a_{2}+\frac{1}{\gamma} a_{2}^{+} a_{2}\right)\right],
$$

tends to zero as $\gamma \rightarrow \infty$.

Although the full role of the closed algebra (2.10) has to be better understood, it is noteworthy that the auxiliary operators $Z$ and $Y$ (see (2.9)) can be interpreted as symmetry variables of the equations

$$
\begin{gathered}
u_{x x}+\epsilon u_{x}-\frac{2}{\gamma}\left(1+\frac{u}{\gamma}\right)^{-2} u_{x}^{2}=-\left(1+\frac{u}{\gamma}\right)^{2}\left[\left(1+\frac{u}{\gamma}\right)^{1-\gamma}\right]_{t t} \\
u_{x x}+\epsilon u_{x}-\frac{3}{2 \gamma}\left(1+\frac{u}{\gamma}\right)^{-1} u_{x}^{2}=-2\left(1+\frac{u}{\gamma}\right)^{\frac{3}{2}}\left[\left(1+\frac{u}{\gamma}\right)^{\frac{1-\gamma}{2}}\right]_{t t},
\end{gathered}
$$

respectively.

Equations (2.19) and (2.20) arise formally from Eq.(1.1) via the transformations

$$
x \rightarrow t, \quad u \rightarrow-\frac{u}{1+\frac{u}{\gamma}}
$$

and

$$
x \rightarrow t, \quad u \rightarrow \gamma\left[\left(1+\frac{u}{\gamma}\right)^{-1 / 2}-1\right] .
$$

From $Z$ we obtain the invariants $x^{\prime}=x, \eta^{\prime}\left(x^{\prime}\right)=\eta(x)$, with

$$
\eta(x)=\left(1+\frac{u}{\gamma}\right)^{\frac{\gamma-2}{2}} t,
$$

which gives the reduced equation

$$
\eta_{x}^{2}+\frac{2-\gamma}{\gamma} \eta \eta_{x x}+\frac{2-\gamma}{\gamma} \epsilon \eta \eta_{x}=\frac{\gamma-1}{\gamma}
$$

from substitution into (2.19). We notice that Eq.(2.24) coincides, formally, with Eq.(3.3) for $x \rightarrow t$ (see Sec. 3). Therefore, putting $\eta=e^{W}$, Eq.(2.24) becomes Eq.(3.4) where $t$ is replaced by $x$. 
On the other hand, the operator $Y$ yields the invariants $x^{\prime}=x, r^{\prime}\left(x^{\prime}\right)=r(x)$, with

$$
r(x)=\left(1+\frac{u}{\gamma}\right)^{\frac{\gamma-2}{2}} t
$$

which leads to the ordinary differential equation of the modified Liouville type (see (3.4))

$$
v_{x x}+\epsilon v_{x}+\frac{1}{\gamma-2} v_{x}^{2}=\frac{2}{\gamma-2}\left[\frac{1}{\gamma}-2(1+\gamma)\right] e^{-2 v},
$$

where $v=\ln r$.

We point out that in the continuous Toda case, i.e. for $\gamma \rightarrow \infty$, in the reduced equations of the one-dimensional modified Liouville type $[(3.4),(3.4)$ written in the variable $x$ and (2.26)], the terms $W_{t}^{2}, W_{x}^{2}$, and $v_{x}^{2}$ disappear. This is due to the fact that for $\gamma \rightarrow \infty$, the coefficient in front of $u \partial_{u}$ in $X_{4}^{0}$ is vanishing, so that $X_{4}^{0}$ is not longer a scale symmetry in the variables $x, u$ (together with a translation of

$\frac{2 \gamma}{\gamma-2}$ along $u$ ). Thus, the presence of square first derivatives in the reduced equations generated by $X_{4}^{0}$ reflects the existence of a scale symmetry, which is broken in the limit $\gamma \rightarrow \infty$.

\section{Explicit solutions}

Here we shall display some significant examples of exact solutions to Eq.(1.1) by using the symmetry approach. To this aim, first let us deal with the generator $X_{4}^{0}$ which appears in both the cases $\epsilon=0$ and $\epsilon \neq 0$. The group transformations involved by $X_{4}^{0}$ are

$$
\begin{aligned}
x^{\prime} & =e^{\lambda} x, \\
t^{\prime} & =t \\
u^{\prime} & =\gamma\left[\left(1+\frac{u}{\gamma}\right) e^{\frac{2 \lambda}{\gamma-2}}-1\right],
\end{aligned}
$$

where $\lambda$ is the group parameter. A set of basis invariants corresponding to (3.1) is given by

$$
\begin{aligned}
& I_{1}=\left(1+\frac{u^{\prime}}{\gamma}\right)^{\frac{2-\gamma}{2}} x^{\prime}=\left(1+\frac{u}{\gamma}\right)^{\frac{2-\gamma}{2}} x \\
& I_{2}=t^{\prime}=t
\end{aligned}
$$

Now, by putting $\rho(t)=I_{1}$, from Eq.(1.1) we obtain the reduced equation

$$
\rho_{t}^{2}+\frac{2-\gamma}{\gamma} \rho \rho_{t t}+\frac{2-\gamma}{\gamma} \epsilon \rho \rho_{t}=\frac{\gamma-1}{\gamma} .
$$


Through the change of variable $\rho=e^{W}$, Eq.(3.3) becomes

$$
W_{t t}+\epsilon W_{t}+\frac{2}{2-\gamma} W_{t}^{2}=\frac{\gamma-1}{2-\gamma} e^{-2 W}
$$

which is a kind of modified one-dimensional Liouville equation. For $\epsilon=0$, Eq.(3.4) can be solved exactly. This occurs via the position

$$
W=\frac{2-\gamma}{2} \ln \theta
$$

which transforms Eq.(3.4) into

$$
\theta_{t t}=\frac{2(\gamma-1)}{(2-\gamma)^{2}} \theta^{\gamma-1}
$$

From (3.6) one easily finds

$$
\theta_{t}^{2}=\frac{4(\gamma-1)}{(2-\gamma)^{2} \gamma} \theta^{\gamma}+c
$$

where $c$ is a constant of integration.

Putting

$$
y^{2}=1+a \theta^{\gamma}
$$

with

$$
a=\frac{4(\gamma-1)}{c \gamma(\gamma-2)^{2}}
$$

Eq.(3.7) provides

$$
\int d y\left(y^{2}-1\right)^{\frac{1-\gamma}{\gamma}}=\frac{\gamma}{2} a^{\frac{1}{\gamma}} \sqrt{c} t+\text { const }
$$

with $c>0$.

In some situations (see Case iv), it may be convenient to write formula (3.10) as

$$
\int(\cosh z)^{-\frac{2}{\gamma}} d z=(-1)^{\frac{\gamma}{1-\gamma}} \frac{\gamma}{2} a^{\frac{1}{\gamma}} \sqrt{c} t+\text { const }
$$

which follows from (3.10) via the change of variable $y=\tanh z$.

Once the integral at the left-hand side of (3.10) has been calculated, one obtains the exact solution

$$
u=\gamma\left[x^{\frac{2}{\gamma-2}}\left(\frac{y^{2}-1}{a}\right)^{\frac{1}{\gamma}}-1\right]
$$

to Eq.(1.1) $(\epsilon=0)$ [see $(3.2 \mathrm{a})$, (3.5), and (3.8)].

At this point, by way of example, we would like to deal with some particular value of the parameter $\gamma$ which is compatible with an explicit expression of $y$ in terms of $t$. 
In what follows we shall deal with the cases $i) \gamma=\frac{1}{2}$, ii) $\gamma=3$, and $\left.i i i\right) \gamma=-2$.

The last two choices correspond, respectively, to the Fermi-Pasta-Ulam potential [3], and to a potential whose nonlinear part, $1 /\left(1-\frac{u}{2}\right)^{2}$, mimics the inverse square potential appearing in the treatment of the scattering states in conformally invariant Quantum Mechanics [12].

Case i) For $\gamma=\frac{1}{2}$, from (3.10) we have

$$
\int\left(y^{2}-1\right) d y=\frac{1}{4} a^{2} \sqrt{c} t
$$

with $a=-\frac{16}{9 c}$, where the constant at the right-hand side of (3.10) has been put equal to zero.

Eq.(3.13) yields the cubic equation

$$
y^{3}-3 y-\frac{3}{4} a^{2} \sqrt{c} t=0
$$

which affords the real solution ([13], p. 17)

$$
y=s_{1}+s_{2}
$$

where

$$
\begin{aligned}
& s_{1}=\left(\frac{3}{8} a^{2} \sqrt{c} t+\sqrt{\frac{9}{64} a^{4} c t^{2}-1}\right)^{\frac{1}{3}}, \\
& s_{2}=\left(\frac{3}{8} a^{2} \sqrt{c} t-\sqrt{\frac{9}{64} a^{4} c t^{2}-1}\right)^{\frac{1}{3}},
\end{aligned}
$$

for $t>\frac{8}{3 a^{2} \sqrt{c}}$.

The remaining solutions of (3.14) are complex conjugate functions, and are given by

$$
y_{1}=-\frac{1}{2}\left(s_{1}+s_{2}\right)+i \frac{\sqrt{3}}{2}\left(s_{1}-s_{2}\right), \quad y_{2}=y_{1}^{*} .
$$

Now, by inserting (3.17) in (3.14) for $\gamma=\frac{1}{2}$ we find the exact solution

$$
u=\frac{1}{2}\left[\frac{1}{a^{2}} x^{-\frac{4}{3}}\left(s_{1}^{2}+s_{2}^{2}+1\right)-1\right] .
$$

Case ii) For $\gamma=3$, Eq.(3.7) provides

$$
\theta_{t}^{2}=\frac{8}{3} \theta^{3}+c
$$


which can be written as

$$
\theta_{\tau}^{2}=4 \theta^{3}-g_{3}
$$

via the rescaling $\tau=\sqrt{\frac{2}{3}} t$, with $g_{3}=-c / 2$. Equation (3.20) is a special version of the equation

$$
\theta_{\tau}^{2}=4 \theta^{3}-g_{2} \theta-g_{3}
$$

which is satisfied by the Weierstrass elliptic function $\mathcal{P}\left(\tau ; g_{2}, g_{3}\right)$, where $g_{2}$ and $g_{3}$ are the invariants of $\mathcal{P}$ (see [13], p. 918).

Concerning Eq. (3.20) we shall distinguish two cases, $g_{3}=0$ and $g_{3} \neq 0$. For $g_{3}=0$, we have

$$
\theta(\tau)=\mathcal{P}(\tau ; 0,0)=\frac{1}{\tau^{2}}
$$

which coincides with the first term of the series representation of $\mathcal{P}\left(\tau ; g_{2}, g_{3}\right)$.

Then, keeping in mind (3.8) and (3.14), we find

$$
u=3\left(\frac{3}{2} \frac{x^{2}}{t^{2}}-1\right) .
$$

For $g_{3} \neq 0$, Eq.(3.20) gives

$$
\int_{\infty}^{\theta} \frac{d \alpha}{\sqrt{4 \alpha^{3}-g_{3}}}=\tau
$$

that is to say

$$
\theta=\mathcal{P}\left(\tau ; 0, g_{3}\right)
$$

Hence, from (3.12) we deduce

$$
u=3\left(x^{2} \theta-1\right)=3\left[x^{2} \mathcal{P}\left(\tau ; 0, g_{3}\right)-1\right] .
$$

We notice that for $c=-2 \quad\left(g_{3}=1\right)$, the Weierstrass function in (3.26) reduces to the equianharmonic case $\mathcal{P}(\tau ; 0,1)([13]$, p. 652).

Case iii) If $\gamma=-2$, from (3.10) we easily obtain

$$
u=-2\left(\sqrt{\frac{c t^{2}-a}{x}}-1\right)
$$

with $a=\frac{3}{8 c}$.

Case iv) For $\gamma=-1$, the potential (1.2) takes the form $\phi \sim(1-u)^{-1}-(1+u)$, which resembles a special case of the generalized Killingbeck potential $V=-\frac{A}{r}+$ $\mathrm{Br}+\mathrm{Cr}^{2}$ [14]. We remind the reader that a Coulomb potential perturbed by a linear term describes the spherical Stark effect in hydrogen [15]. Putting in (3.11) $\gamma=-1$ and $c=-|c|$, we arrive at the relation

$$
2 z+\sinh 2 z=\frac{9}{4}|c|^{\frac{3}{2}} t+\text { const }
$$


Curiously enough, by replacing formally the hyperbolic sine by the exponential, Eq.(3.28) becomes an equation of the Schroeder type, which appears in the bootstrap model and in renormalization theory, whose analytical structure was investigated by Hagedorn and Rafelsky [16]. These authors obtained a solution of the Schroeder equation both as a power series expansion and as an integral representation. Thus, it should be of interest to try to adopt the same strategy in the study of Eq.(3.28).

Another nontrivial example of exact solution to Eq.(1.1) can be determined starting from the linear combination of $X_{3} \equiv X_{3}^{0}=\partial_{x}$ and $X_{2} \equiv X_{2}^{0}=\partial_{t}$ :

$$
V=v \partial_{x}+\partial_{t}
$$

where $v$ is a (real) constant. The group transformations are $x^{\prime}=x+v \lambda, \quad t^{\prime}=t+\lambda$, which provide the invariant $\xi=x^{\prime}-v t^{\prime}=x-v t$. Thus, by inserting the variable $\xi$ into Eq.(1.1), we get the ordinary differential equation

$$
v^{2} u_{\xi \xi}+\epsilon v u_{\xi}=\left[\left(1+\frac{u}{\gamma}\right)^{\gamma-1}\right]_{\xi \xi}
$$

which gives

$$
v^{2} u_{\xi}-\epsilon v u=\left[\left(1+\frac{u}{\gamma}\right)^{\gamma-1}\right]_{\xi}+c_{0},
$$

where $c_{0}$ is a constant of integration. By choosing $c_{0}=0, v^{2}=\frac{\gamma-1}{\gamma}$ and limiting ourselves to consider those values of $\gamma$ such that $\gamma=-|\gamma|$, Eq.(3.31) yields

$$
\left[1-\left(1-\frac{u}{|\gamma|}\right)^{-|\gamma|-2}\right]_{\xi}=\frac{\epsilon}{v} u
$$

from which

$$
\int_{0}^{u} \frac{1}{u^{\prime}}\left[1-\left(1+\frac{u^{\prime}}{|\gamma|}\right)^{-|\gamma|-2}\right] d u^{\prime}=\frac{\epsilon}{v}\left(\xi-\xi_{0}\right),
$$

$\xi_{0}$ being a constant of integration.

We notice that for $|\gamma| \rightarrow \infty$, Eq.(3.33) becomes

$$
\int_{0}^{u} \frac{1-e^{-u^{\prime}}}{u^{\prime}} d u^{\prime} \equiv \operatorname{Ein}(u)=\frac{\epsilon}{v}\left(\xi-\xi_{0}\right)
$$

where $\operatorname{Ein}(u)$ denotes the exponential-integral function ([17], p. 255)

$$
\operatorname{Ein}(u)=-E i(-u)+\ln u+C,
$$


$E i(-u)=-\int_{u}^{\infty} \frac{e^{-u^{\prime}}}{u^{\prime}} d u^{\prime}$, and $C$ is the Euler-Mascheroni constant, defined by [17]

$$
C=-\psi(1)=-\int_{0}^{\infty} e^{-t} \ln t d t
$$

where $\psi(z)=\frac{\Gamma^{\prime}(z)}{\Gamma(z)}$ is the psi (or digamma) function ([13], p. 258).

At this point, let us introduce the function

$$
\begin{aligned}
\hat{\Gamma}(\alpha, u ;|\gamma|) & =\int_{u}^{\infty}\left(1+\frac{u^{\prime}}{|\gamma|}\right)^{-(2+|\gamma|)}\left(u^{\prime}\right)^{\alpha-1} d u^{\prime}= \\
& =\hat{\Gamma}(\alpha, 0 ;|\gamma|)-\int_{0}^{u}\left(1+\frac{u^{\prime}}{|\gamma|}\right)^{-(2+|\gamma|)}\left(u^{\prime}\right)^{\alpha-1} d u^{\prime}
\end{aligned}
$$

which holds for $0<\alpha<2+|\gamma|$.

We point out that for $|\gamma| \rightarrow \infty, \hat{\Gamma}(\alpha, u ;|\gamma|)$ reproduces the incomplete gammafunction $\Gamma(\alpha, x)(13$ p. 260).

Now, since the integral at the right-hand site of (3.37) can be written as [17]

$$
\begin{aligned}
\int_{0}^{u}\left(u^{\prime}\right)^{\alpha-1}\left(1+\frac{u^{\prime}}{|\gamma|}\right)^{-(2+|\gamma|)} d u^{\prime} & =\frac{u^{\alpha}}{\alpha}{ }_{2} F_{1}\left(2+|\gamma|, \alpha ; \alpha+1,-\frac{u}{|\gamma|}\right)= \\
& =\frac{u^{\alpha}}{\Gamma(2+|\gamma|)} \sum_{n=0}^{\infty} \frac{\Gamma(2+|\gamma|+n)}{(\alpha+n) n !}(-1)^{n}\left(\frac{u^{\prime}}{|\gamma|}\right)^{n}
\end{aligned}
$$

Eq.(3.37) takes the form

$$
\begin{aligned}
\hat{\Gamma}(\alpha, u ;|\gamma|)= & \hat{\Gamma}(\alpha, 0,|\gamma|)-\frac{u^{\alpha}}{\alpha}- \\
& \frac{u^{\alpha}}{\Gamma(2+|\gamma|)} \sum_{n=1}^{\infty} \frac{\Gamma(2+|\gamma|+n)}{(\alpha+n) n !}(-1)^{n}\left(\frac{u}{|\gamma|}\right)^{n}
\end{aligned}
$$

where $\Gamma(\cdot)$ is the gamma-function and ${ }_{2} F_{1}$ is the the Gauss hypergeometric series, respectively $(13$, p. 556).

We have (see [17], p. 209)

$$
\begin{aligned}
& \lim _{\alpha \rightarrow 0} \hat{\Gamma}(\alpha, u ;|\gamma|)= \\
& =\lim _{\alpha \rightarrow 0}\left[\frac{\alpha \hat{\Gamma}(\alpha, 0 ;|\gamma|)-u^{\alpha}}{\alpha}-\frac{u^{\alpha}}{\Gamma(2+|\gamma|)} \sum_{n=1}^{\infty} \frac{\Gamma(2+|\gamma|+n)}{(\alpha+n) n !}(-1)^{n}\left(\frac{u}{|\gamma|}\right)^{n}\right]= \\
& =-\ln u+\ln |\gamma|-\psi(2+|\gamma|)-C-\int_{0}^{u} \frac{1}{u^{\prime}}\left[\left(1+\frac{u^{\prime}}{|\gamma|}\right)^{-(2+|\gamma|)}-1\right] d u^{\prime}
\end{aligned}
$$


On the other hand, from (3.37) we obtain (see [13 p.255)

$$
\lim _{\alpha \rightarrow 0} \hat{\Gamma}(\alpha, u ;|\gamma|)=\int_{u}^{\infty}\left(1+\frac{u^{\prime}}{|\gamma|}\right)^{-(2+|\gamma|)} \frac{1}{u^{\prime}} d u^{\prime}
$$

Finally, by comparing (3.41) with (3.40), we find

$$
\begin{aligned}
\int_{u}^{\infty}\left(1+\frac{u^{\prime}}{|\gamma|}\right)^{-(2+|\gamma|)} \frac{1}{u^{\prime}} d u^{\prime}= & \ln \frac{|\gamma|}{u}-\psi(2+|\gamma|)-C+ \\
& \int_{0}^{u} \frac{1}{u^{\prime}}\left[1-\left(1+\frac{u^{\prime}}{|\gamma|}\right)^{-(2+|\gamma|)}\right] d u^{\prime}
\end{aligned}
$$

that is (see (3.33))

$$
\begin{aligned}
\hat{\Gamma}(0, u ;|\gamma|) & =\int_{u}^{\infty}\left(1+\frac{u^{\prime}}{|\gamma|}\right)^{-(2+|\gamma|)} \frac{1}{u^{\prime}} d u^{\prime}= \\
& =\frac{\epsilon}{v}\left(\xi-\xi_{0}\right)+\ln \frac{|\gamma|}{u}-\psi(2+|\gamma|)-C .
\end{aligned}
$$

We remark that $\lim _{|\gamma| \rightarrow \infty}[\ln |\gamma|-\psi(2+|\gamma|)]=0$ ([18], p. 945).

Therefore, Eq. (3.43) becomes

$$
\lim _{|\gamma| \rightarrow \infty} \hat{\Gamma}(0, u ;|\gamma|)=-E i(-u)=\frac{\epsilon}{v}\left(\xi-\xi_{0}\right)-\ln u-C,
$$

from which (see (3.35))

$$
\operatorname{Ein}(u)=\frac{\epsilon}{v}\left(\xi-\xi_{0}\right)
$$

This result, corresponding to the continuous Toda case, has been already obtained in [10]. Consequently, the function $\hat{\Gamma}(\alpha, u ;|\gamma|)$ defined by (3.37) can be considered as an extended version of the incomplete gamma function $\Gamma(\alpha, u)=\int_{u}^{\infty} e^{-t} t^{\alpha-1} d t$ (see [13] p. 260).

The quantity (3.43), where $\hat{\Gamma}(0, u ;|\gamma|)$ can be interpreted as a generalization of the exponential-integral function, constitutes an implicit solution of Eq.(1.1).

\section{Linearization and algebraic properties}

Equation (1.1) can be handled within the prolongation scheme [9]. In doing so, let us consider the prolongation equations for Eq.(1.1):

$$
y_{x}^{i}=F^{i}\left(u, u_{t} ; y\right), \quad y_{t}^{i}=G^{i}\left(u, u_{x_{j}} ; y\right),
$$


where $i=1,2, \ldots N$ ( $N$ arbitrary), and the set of variables $y \equiv\left\{y^{i}\right\}$ is the pseudopotential [9] $(j=1,2, \ldots M(M$ arbitrary $))$.

The compatibility condition for Eqs. (4.1) gives

$$
\begin{aligned}
F^{i} & =\frac{\gamma}{\gamma-1} L^{i}(y) u_{t}+M^{i}(u, y) \\
G^{i} & =L^{i}(y)\left(1+\frac{u}{\gamma}\right)^{\gamma-2} u_{x}+P^{i}(u ; y),
\end{aligned}
$$

where $M^{i}=M^{i}(u ; y), P^{i}=P^{i}(u ; y)$ and $L^{i}=L^{i}(y)$ are defined by

$$
\begin{aligned}
\frac{\gamma-1}{\gamma} M_{u}^{i}+[P, L]^{i} & =\epsilon L^{i}, \\
\left(1+\frac{u}{\gamma}\right)^{\gamma-2}[L, M]^{i} & =P_{u}^{i}, \\
{[M, P]^{i} } & =0,
\end{aligned}
$$

with $[P, L]^{i}=P^{k} \frac{\partial L^{i}}{\partial y^{k}}-L^{k} \frac{\partial P^{i}}{\partial y^{k}}$, and so on.

In order to explore the prolongation equations (4.3), for brevity we shall omit the index $i$.

It is noteworthy the following

Proposition 3 Let $u$ be a solution of Eq.(1.5). Then, the function $y_{2}=y_{2}(x, t)$ defined by

$$
\begin{gathered}
y_{2 x}=W(u) \sinh y_{1}, \quad y_{2 t}=S(u) \cosh y_{1}, \\
y_{1 x}=\frac{\gamma}{\gamma-1} u_{t}, \quad y_{1 t}=\left(1+\frac{u}{\gamma}\right)^{\gamma-2} u_{x},
\end{gathered}
$$

satisfies the wave equation

$$
y_{2 t t}=\frac{\gamma-1}{\gamma}\left(1+\frac{u}{\gamma}\right)^{\gamma-2} y_{2 x x}
$$

where $W(u)$ and $S(u)$ obey the linear differential equations of the Bessel type

$$
W_{u u}=\frac{\gamma}{\gamma-1}\left(1+\frac{u}{\gamma}\right)^{\gamma-2} W
$$

and

$$
S_{u u}=\frac{\gamma}{\gamma-1}\left(1+\frac{u}{\gamma}\right)^{\gamma-2} S+\frac{\gamma-2}{\gamma}\left(1+\frac{u}{\gamma}\right)^{-1} S_{u} .
$$


Equation (4.6) represents a linearized version of Eq.(1.5).

To prove this Proposition, let us look for a solution to Eqs.(4.3) of the form

$$
M=W(u) V(y), \quad P=S(u) T(y) .
$$

Then Eqs. (4.3) provide

$$
S=\frac{\gamma-1}{\gamma} W_{u}, \quad S_{u}=\left(1+\frac{u}{\gamma}\right)^{\gamma-2} W
$$

and

$$
\left[X_{1}, X_{2}\right]=X_{3}, \quad\left[X_{1}, X_{3}\right]=X_{2}, \quad\left[X_{2}, X_{3}\right]=0 .
$$

with $L \equiv X_{1}, V \equiv X_{2}, T \equiv X_{3}$.

Equations (4.10) imply equations (4.7) and (4.8). By means of the change of variable

$$
z=2 i \sqrt{\frac{\gamma}{\gamma-1}}\left(1+\frac{u}{\gamma}\right)^{\frac{\gamma}{2}},
$$

Eq. (4.7) is transformed into the Bessel equation

$$
z^{2} Z_{z z}+z Z_{z}+\left(z^{2}-\frac{1}{\gamma^{2}}\right) Z=0,
$$

where $Z=Z(z)$ is related to $W$ by

$$
W=\left(1+\frac{u}{\gamma}\right)^{\frac{1}{2}} Z\left[2 i \sqrt{\frac{\gamma}{\gamma-1}}\left(1+\frac{u}{\gamma}\right)^{\frac{\gamma}{2}}\right] .
$$

On the other hand, Eq. (4.8) takes the form

$$
z^{2} Z_{z z}+z Z_{z}+\left[z^{2}-\left(\frac{\gamma-1}{\gamma}\right)^{2}\right] Z=0,
$$

where

$$
S=\left(1+\frac{u}{\gamma}\right)^{\frac{\gamma-1}{2}} Z\left[2 i \sqrt{\frac{\gamma}{\gamma-1}}\left(1+\frac{u}{\gamma}\right)^{\frac{\gamma}{2}}\right] .
$$

$Z$ stands for a generic Bessel function of index $\pm \frac{1}{\gamma}$ and $\pm \frac{\gamma-1}{\gamma}$, respectively ([13], pag. 358). 
The commutation rules (4.11) define the Lie algebra corresponding to the Euclidean group $E_{2}$ in the plane. A realization of (4.11) in terms of a two component pseudopotential $y \equiv\left(y_{1}, y_{2}\right)$ is

$$
X_{1}=\partial_{y_{1}}, \quad X_{2}=\sinh y_{1} \partial_{y_{2}}, \quad X_{3}=\cosh y_{1} \partial_{y_{2}} .
$$

Therefore, with the help of (4.2a and (4.2b), Eqs. (4.1) take the form expressed by (4.4) and (4.5). Furthermore, by differentiating $y_{2 x}$ with respect to $x$ and $y_{2 t}$ with respect to $t$ (see (4.4) ) and using (4.5) and (4.10), one arrives at the wave equation (4.6).

Finally, we observe that for $\gamma=3$, i.e. in the case of the Fermi-Pasta-Ulam potential, Eq. (4.7) is led to the Airy equation (413, pag. 446).

$$
W_{\sigma \sigma}=\sigma W
$$

via the transformation $\sigma=2^{-\frac{1}{3}}(u+3)$.

The prolongation equations (4.3) offer the possibility of getting a further insight into the algebraic structure of Eq.(1.1).

To this aim, let us write Eqs.(4.3) in the form

$$
\begin{aligned}
& \frac{\gamma-1}{\gamma} P_{z}+[M, L]=0, \quad \frac{\gamma-1}{\gamma} z^{\frac{\gamma-2}{\gamma-1}} M_{z}=\epsilon L+[L, P], \\
& {[M, P]=0,}
\end{aligned}
$$

where $z=\left(1+\frac{u}{\gamma}\right)^{\gamma-1}$. Then, we look for a solution to Eqs.4.19) such that

$$
M=\sum_{k=0}^{\infty} a_{k}(y) z^{k}, \quad P=\sum_{k=0}^{\infty} b_{k}(y) z^{k} .
$$

In the following, we limit ourselves to characterize mainly the algebraic structure of Eq.(1.1) for a particular sequel of values of the parameter $\gamma$ (see Proposition 4). In this case it turns out that a finite-dimensional Lie algebra is associated with Eq.(1.1). This algebra is used to write Eq.(1.1) in a potential form, which allows us to estabilish some analogies between the prolongation and the symmetry approaches. In general, namely for any value of $\gamma$ (provided that $\gamma \neq 0,1$ ) a systematic analysis of the algebraic properties of Eq.(1.1), which is based on the ansatz (5.2), requires further efforts.

Substitution from (4.20) into Eq.(4.19) gives the following constraints between the coefficients $a_{k}(y)$ and $b_{k}(y)$ :

$$
\begin{aligned}
& k \frac{\gamma-1}{\gamma} b_{k}+\left[a_{k-1}, L\right]=0, \\
& \frac{\gamma-1}{\gamma}\left(a_{1}+2 a_{2} z+3 a_{3} z^{2}+4 a_{4} z^{3}+\ldots\right) z^{\frac{\gamma-2}{\gamma-1}}= \\
& =\epsilon L+\left[L, b_{0}+b_{1} z+b_{2} z^{2}+b_{3} z^{3}+\ldots\right]
\end{aligned}
$$


and

$$
\begin{aligned}
& {\left[a_{0}, b_{0}\right]=0,} \\
& {\left[a_{0}, b_{1}\right]+\left[a_{1}, b_{0}\right]=0,} \\
& {\left[a_{0}, b_{2}\right]+\left[a_{1}, b_{1}\right]+\left[a_{2}, b_{0}\right]=0,} \\
& \ldots \ldots \ldots \ldots \ldots \ldots \\
& \sum_{k=1}^{N}\left[a_{k-1}, b_{N-k}\right]=0,
\end{aligned}
$$

with $N$ arbitrary.

The following property holds

Proposition 4 If $\epsilon \neq 0$ and $\nu=\frac{\gamma-2}{\gamma-1}$ is such that $\nu \neq \ldots,-3,-2,-1,0,1,2,3 \ldots$, Eqs.(4.21) imply the finite-dimensional Lie algebra $\mathcal{L}$

$$
\begin{aligned}
{\left[a_{0}, b_{0}\right] } & =\left[a_{0}, b_{1}\right]=\left[L, b_{1}\right]=0, \\
{\left[b_{0}, b_{1}\right] } & =\epsilon b_{1}, \\
{\left[b_{0}, L\right] } & =\epsilon L, \\
{\left[a_{0}, L\right] } & =\frac{1}{\nu-2} b_{1},
\end{aligned}
$$

for $\epsilon \neq 0$, and the finite-dimensional Lie algebra $\mathcal{L}^{\prime}$

$$
\begin{aligned}
& {\left[a_{0}, b_{0}\right]=\left[a_{0}, b_{1}\right]=\left[L, b_{1}\right]=\left[b_{0}, b_{1}\right]=\left[b_{0}, L\right]=0,} \\
& {\left[a_{0}, L\right]=\frac{1}{\nu-2} b_{1},}
\end{aligned}
$$

for $\epsilon=0$.

The proof is straightforward. In fact, under the assumption that $\nu \neq \ldots$, $-3,-2,-1, \ldots$, from (4.21b) we obtain $a_{k}=0,\left[b_{k}, L\right]=0 \quad(k=1,2,3, \ldots)$, and the commutation relation (4.220). Furthermore, Eq.4.21c entails the commutation relation (4.22d ) and $b_{2}=b_{3}=\ldots=0$. Furthermore, Eq. (4.22b) can be determined by elaborating Eq.(4.22d) via the Jacobi identity applied to $\left[a_{0},\left[b_{0}, L\right]\right]$. The commutation relations (4.23) emerge immediately.

A matrix representation of $\mathcal{L}$ is

$$
a_{0}=\left(\begin{array}{ccc}
0 & -1 & 0 \\
0 & 0 & 0 \\
0 & 0 & 0
\end{array}\right), \quad b_{0}=\left(\begin{array}{ccc}
\epsilon & 0 & 0 \\
0 & \epsilon & 0 \\
0 & 0 & 0
\end{array}\right)
$$




$$
b_{1}=\left(\begin{array}{ccc}
0 & 0 & 1 \\
0 & 0 & 0 \\
0 & 0 & 0
\end{array}\right), \quad L=\left(\begin{array}{ccc}
0 & 0 & 0 \\
0 & 0 & \frac{1}{2-\nu} \\
0 & 0 & 0
\end{array}\right)
$$

Then, Eqs. (4.1) take the form

$$
\begin{aligned}
& \left(\begin{array}{l}
y_{1} \\
y_{2} \\
y_{3}
\end{array}\right)_{x}=\left(\begin{array}{ccc}
0 & 0 & 0 \\
-1 & 0 & 0 \\
0 & u_{t} & 0
\end{array}\right)\left(\begin{array}{l}
y_{1} \\
y_{2} \\
y_{3}
\end{array}\right), \\
& \left(\begin{array}{l}
y_{1} \\
y_{2} \\
y_{3}
\end{array}\right)_{t}=\left(\begin{array}{ccc}
\epsilon & 0 & 0 \\
0 & \epsilon & 0 \\
\left(1+\frac{u}{\gamma}\right)^{\gamma-1} & \frac{\gamma-1}{\gamma} u_{x}\left(1+\frac{u}{\gamma}\right)^{\gamma-2} & 0
\end{array}\right)\left(\begin{array}{l}
y_{1} \\
y_{2} \\
y_{3}
\end{array}\right),
\end{aligned}
$$

from which $y_{1}=\lambda_{1} e^{\epsilon t}, y_{2}=\left(\lambda_{0}-\lambda_{1} x\right) e^{\epsilon t}$, and

$$
\begin{aligned}
& y_{3 \zeta}=-\frac{1}{\lambda_{1}} e^{\epsilon t} u_{t} \zeta \\
& y_{3 t}=-\lambda_{1} e^{\epsilon t} \zeta^{2} \frac{\partial}{\partial \zeta} \frac{\left(1+\frac{u}{\gamma}\right)^{\gamma-1}}{\zeta},
\end{aligned}
$$

where $\zeta=\lambda_{0}-\lambda_{1} x$, and $\lambda_{0}, \lambda_{1}$ are constants of integration. Here $y_{3}$ can be interpreted as a potential variable.

Equations (4.25a) and (4.25b) allow us to find, in theory, special explicit solutions to Eq.(1.1). For example, let us assume that $u_{t}=\beta(t) \zeta^{\frac{2}{\gamma-2}}$. Then, Eqs. (4.26a) and (4.26b) give rise to the differential equation for $\beta(t)$ :

$$
\beta=\gamma b^{\frac{1}{\gamma-1}} \frac{d}{d t}(\dot{\beta}+\epsilon \beta)^{\frac{1}{\gamma-1}}
$$

where $b=\frac{(\gamma-2)^{2}}{2 \gamma(\gamma-1)} \lambda_{1}{ }^{2}$.

Equation (4.27) can be written as

$$
\ddot{V}+\epsilon \dot{V}=k V^{\gamma-1}
$$

$\left(k=\frac{b^{-\frac{1}{\gamma-1}}}{\gamma}\right)$ through the position

$$
\dot{\beta}+\epsilon \beta=V^{\gamma-1} \text {. }
$$

For $\epsilon=0$, we may use for the algebra $\mathcal{L}^{\prime}$ the same representation (5.6), where now $b_{0}$ is the null matrix. In this case Eq. (4.28) coincides with Eq.(3.6), which leads to explicit solutions of the original equation (1.5) for special values of the parameter 
$\gamma$ (see Sect.3). We remark that via the change of variable $V=e^{\frac{2}{2-\gamma} W}$ and by a suitable choice of the free constant $k$, Eq.(4.28) becomes Eq.(3.4), which arises from the symmetry operator $X_{4}{ }^{0}$ in the context of the Lie group theory. This fact suggests the existence of a possible link between the prolongation method and the symmetry approach. However, our result is indicative only, and many aspects of the problem remain to be elucidated.

\section{Comments}

We have dealt with a nonlinear field equation arising as the continuous limit of a lattice model containing many cases of physical significance (the harmonic, Toda, Fermi-Pasta-Ulam, Coulomb-like lattices, and others). This equation, which is new at the best of our knowledge, can be considered as a paradigm for the application of different analytical procedures. The addition of a perturbative friction-like term has allowed us to check the limits of validity of these methods. These are the symmetry and approximate symmetry approach, and the prolongation technique. The joint resort to these methods leads to the discovery of some interesting properties of Eq.(1.1), which have been expounded in the Introduction.

The spirit of this paper is both of methodological and speculative character. The results obtained are a challange to apply the same strategy to investigate a threedimensional version of Eq.(1.1). This purpose might be important in nonlinear field theories based on the deformation of the algebra used in the study of dispersionless field equations [19]. 


\section{Bibliography}

[1] M. Leo, R. A. Leo and G. Soliani, Phys. Lett. A60 (1977) 283.

[2] M. Toda, Theory of nonlinear lattices, in Solid-State Science, vol. 20 (Springer, Berlin, 1981).

[3] E. Fermi, J. Pasta and S. Ulam, Los Alamos Report LA-1940, in Collected papers of Enrico Fermi, edited by E. Segre (University of Chicago, 1965), Vol.2, p.978.

[4] See, for example, M. V. Saveliev, Theor. Math. Fiz. 92 (1992) 457, and references therein.

[5] J. D. Finley and J. F. Plebanski, J. Math. Phys. 20 (1979) 1938.

[6] C. P. Boyer and J. D. Finley, J. Math. Phys. 23 (1982) 1126.

[7] P. J. Olver, Applications of Lie Groups to Differential Equations (Springer, New York, 1986).

[8] V. A. Baikov, R. K. Gazizov and N. H. Ibragimov, Mat. Sbornik 136 (No. 4) (1988) 435 [English transl. in Math. USSR Sbornik 64 (No. 2) (1989) 427].

[9] H. D. Wahlquist and F. B. Estabrook, J. Math. Phys. 16 (1975) 1.

[10] E. Alfinito, G. Profilo and G. Soliani, Properties of equations of the continuous Toda type, Preprint 1996 (Universita‘ di Lecce).

[11] C. Lebrun, J. Diff. Geom. 34 (1991) 223.

[12] V. Barone, V. Penna and P. Sodano, Ann. Phys. 225 (1993) 212.

[13] M. Abramowitz and I. Stegun (Eds.), Handbook of mathematical functions (Dover, New York, 1975).

[14] J. Killinbeck, Phys. Lett. 67A (1978) 13.

[15] S. C. Chhajlarry and D. A. Letov, Phys. Rev. 44A (1991) 4725, and references therein. 
[16] R. Hagedorn and J. Rafelski, Comm. Math. Phys. 83 (1983) 563.

[17] F. G. Tricomi, Funzioni Ipergeometriche Confluenti, (Edizioni Cremonese, Roma, 1954).

[18] I. S. Gradshteyn and I. M. Ryzhik, Table of Integrals Series and Products (Academic Press, New York and London, 1965).

[19] I. A. B. Strachan, J. Phys. A: Math. Gen. 29 (1996) 6117. 